\title{
Comparative analysis of triterpenoids from Mikania cordifolia collected from four different locations
}

\author{
Patrícia Abrão de Oliveira, ${ }^{1}$ Izabel Cristina Casanova Turatti ${ }^{2}$, Dionéia Camilo \\ Rodrigues de Oliveira ${ }^{2 *}$
}

\begin{abstract}
'Departamento de Química, Faculdade Filosofia, Ciências e Letras de Ribeirão Preto, Universidade de São Paulo, 2Departamento de Física e Química, Faculdade de Ciências Farmacêuticas de Ribeirão Preto,

Universidade de São Paulo
\end{abstract}

\section{*Correspondence:}

D. C. R. Oliveira

Faculdade de Ciências Farmacêuticas

de Ribeirão Preto

Departamento de Física e Química

Universidade de São Paulo

Av. do Café, $\mathrm{s} / \mathrm{n}^{\circ}$

14040-903 - Ribeirão Preto - SP - Brasil

E-mail: drolivei@ffrp.usp.br
The species Mikania cordifolia is distributed across America and widely found throughout Brazilian territory, where is popularly used against snake bites. Methanolic and dichloromethanic extracts prepared from M. cordifolia Robinson collected from four different locations in Brazil were submitted to liquid-liquid extraction and the hexanic phase and residues obtained from this step were analyzed for triterpenoids by gas chromatography. The specimens from Ribeirão Preto-SP and São Carlos-SP showed similar triterpenoid composition: Damyrin, lupeol, lupenone, Damyrin acetate, E-amyrin acetate, lupeol acetate, taraxasterol acetate, campesterol and E-sitosterol. Besides these triterpenoids, the specimen from Campos de Jordão-SP presented 11-oxours-12-ene, 11-oxoolean12-ene and taraxerol acetate, and from Monte Verde, epitaraxerol e taraxerol acetate. The triterpene friedelin could be found in specimens from Ribeirão Preto and São Carlos.

\section{INTRODUCTION}

The species Mikania cordifolia Robinson (Asteraceae, Eupatorieae) is widely distributed in America and can be found throughout Brazilian territory. $M$. cordifolia, as well other Mikania species, is known popularly as "guaco" and has been used for treatment of respiratory problems (Caribe et al., 1991). M. cordifolia has an ancient use by rainforests inhabitants to treat snake bites and, due to this application, it has been called "snake vine" (Caribe et al., 1991; Mors et al., 2000). Biological activities described for $M$. cordifolia include antitrichomonal, antitrypanosomal and insecticide activities
(Arias et al., 1995; Serrano et al., 2000). The genus Mikania has been extensively studied and for $M$. cordifolia, it has been described the presence of diterpenes, sesquiterpenes and phenylpropanoids (Boeker et al., 1987; Bohlmann et al., 1978; Castro et al.,1989; D' Agostino et al., 1990; Herz et al., 1977).

In continuation to our study on chemistry of Mikania species (Chaves et al., 2003; Fabbri et al., 1997; Nascimento et al. 2004; Nascimento, Oliveira, 2001; Veneziani, Oliveira, 1999) and considering the importance of this genus in tradicional medicine, we have examined $M$. cordifolia extracts from four different locations in Brazil. 


\section{MATERIAL AND METHODS}

\section{Plant material}

The specimens were collected from four different locations in Brazil (Table I) with two distinct vegetations and were identified by Dr. Roberto Lourenço Esteves (Departamento de Biologia Animal e Vegetal, Universidade Estadual do Rio de Janeiro, Brazil). Voucher specimens were deposited at the Herbarium Bradeanum (Universidade Estadual do Rio de Janeiro) and the Herbarium of FFCLRP (Universidade de São Paulo).

\section{Extraction}

Aerial parts of each specimen $(600 \mathrm{~g})$ were dried, pulverized and macerated with methanol and dichloromethane. The resulting extracts were filtered, concentrated under reduced pressure and dissolved in methanol:water (9:1). These extracts were submitted to liquid-liquid extraction with $n$-hexane. The residue obtained after filtration (R) and the hexanic phase (HP) of each methanolic (ME) and dichloromethanic extract (DE) were submitted to a clean-up step.

\section{Clean-up procedure}

$10 \mathrm{mg}$ of residues $(\mathrm{R})$ and the hexanic phases (HP) of each methanolic (ME) and dichloromethanic extract (DE) of each $M$. cordifolia specimen were percolated though a silica gel column (Alltec, silica gel $200 \mathrm{mg}, 3 \mathrm{~mL}$ ) with $10 \mathrm{~mL}$ of $n$-hexane and $10 \mathrm{~mL}$ of chloroform (Schinor et al., 2004).

\section{Gas chromatography analysis}

Chloroformic fractions were analyzed by high resolution gas chromatography (HRGC) on a HewlettPackard model 5890 Series II gas chromatograph with Flame Ionization Detector (FID) at $330^{\circ} \mathrm{C}$ and a split injector (split ratio $1: 50$ at $260^{\circ} \mathrm{C}$ ). Two capillary columns were employed to perform the analysis: HP-1 (cross-linked methyl-silicone, $30 \mathrm{~m}$ x $0.25 \mathrm{~mm} \times 0.25 \mathrm{Pm}$ ) and HP-50 (cross-linked 50\% phenyl-methyl-silicone $30 \mathrm{~m}$ x $0.25 \mathrm{~mm}$ $\mathrm{x} 0.25 \mathrm{Pm}$ ). For HP-1, the temperature program was $250{ }^{\circ} \mathrm{C}$ held for $12 \mathrm{~min}$, increased $6^{\circ} \mathrm{C} / \mathrm{min}$ to $280^{\circ} \mathrm{C}$, and held this temperature for $30 \mathrm{~min}$ and for $\mathrm{HP}-50$, the program was initiated at $50{ }^{\circ} \mathrm{C}$, increased $20{ }^{\circ} \mathrm{C} / \mathrm{min}$ to $250{ }^{\circ} \mathrm{C}$ (held for $4 \mathrm{~min}$ ), increased $15^{\circ} \mathrm{C} / \mathrm{min}$ to $280^{\circ} \mathrm{C}$ (held for $18 \mathrm{~min}$ ), increased $10^{\circ} \mathrm{C} / \mathrm{min}$ to $290^{\circ} \mathrm{C}$ (held for $30 \mathrm{~min}$ ). All the standard compounds used in these analyses were isolated from different plant material in our laboratory and identified by spectral data with the exception of campesterol, stigmasterol and E-sitosterol (Supelco Inc.). Cholesterol was used as reference standard (Schinor et al., 2004).

\section{RESULTS AND DISCUSSION}

The residues (R) and hexanic phases (HP) were obtained in proportions ranging from $38 \%$ to $60 \%$ for methanolic extracts (ME) and from $76 \%$ to $91 \%$ for dichloromethanic extracts (DE). These great amounts of lipid material in addition to innumerous biological activities described for triterpenoids, such as antitumor, antiinflammatory and antioxidant (Moghadasian, 2000; Osvena et al., 2004; Saleen et al., 2004; Sultana et al., 2003), made the research of triterpenes and steroids relevant. The analysis of triterpenoids in Mikania cordifolia is essential because this group of substances may be related to neutralization of Bothrops jararaca venom (Mors et al., 2000).

In this present work, the results of triterpenoids analysis summarized in Tables II, III, IV and V revealed that the different specimens of $M$. cordifolia, independently of collecting site, are rich in triterpenoids. These compounds were identified by comparison of relative retention value (considering cholesterol value 1.0) and campesterol, stigmasterol, E-sitosterol, E-amyrin, lupenone, lupeol, friedelin, pseudotaraxasterol, taraxasterol, E-amyrin

TABLE I - Collection data of Mikania cordifolia specimens

\begin{tabular}{lccccc}
\hline Location & Coletor Number* & Deposit Number** & Altitude $(\mathrm{m})$ & Vegetation & Date \\
\hline Ribeirão Preto - SP & NPL-259 & SPFR-05313 & 595 & Cerrado & 27 May 2000 \\
São Carlos - SP & NPL-261 & SPFR-05315 & 854 & Cerrado & 27 May 2000 \\
Campos de Jordão - SP & NPL-264 & SPFR-05314 & 1030 & Forest & 29 May 2000 \\
Monte Verde - MG & NPL-266 & SPFR-05316 & 947 & Forest & 29 May 2000 \\
\hline
\end{tabular}

*Herbarium Bradeanum (Universidade Estadual do Rio de Janeiro); **Herbarium of the Faculdade de Filosofia Ciências e Letras de Ribeirão Preto/Universidade de São Paulo 
TABLE II -Triterpenoid composition of M. cordifolia specimen collected from Ribeirão Preto

\begin{tabular}{lcccc}
\hline Compounds & DE-R (\%) & DE-HP (\%) & ME-R (\%) & ME-HP (\%) \\
\hline Campesterol & 0.37 & 0.97 & - & - \\
Stigmasterol & 2.15 & 5.85 & - & 0.33 \\
E-Sitosterol & 2.21 & 4.55 & 0.85 & 1.07 \\
Epitaraxerol & - & - & - & - \\
E-Amyrin & 2.31 & 5.04 & - & 0.31 \\
D-Amyrin/Lupenone & 1.59 & 4.85 & 2.45 & 2.75 \\
Lupeol & 2.93 & 9.96 & 2.10 & 1.87 \\
Taraxerol acetate & - & - & - & - \\
Friedelin & 3.17 & 2.10 & 3.53 & 3.07 \\
Pseudotaraxasterol & 0.38 & 0.27 & 4.21 & 2.67 \\
Taraxasterol & 2.60 & 3.34 & - & 1.66 \\
11-oxours-12-ene & - & - & - & - \\
11-oxoolean-12-ene & - & - & - & - \\
E-Amyrin acetate & 8.24 & 7.36 & 5.70 & 5.00 \\
D-Amyrin acetate & 0.88 & 0.68 & 1.26 & 1.39 \\
Lupeol acetate & 15.72 & 16.38 & 8.93 & 7.51 \\
Taraxasterol acetate & 28.01 & 2.12 & 4.78 & 4.14 \\
\hline
\end{tabular}

DE: dichloromethanic extract; ME: methanolic extract; HP: hexanic phase; R: residue; -: traces.

TABLE III -Triterpenoid composition of M. cordifolia specimen collected from São Carlos

\begin{tabular}{lcccc}
\hline Compounds & DE-R (\%) & DE-HP $(\%)$ & ME-R (\%) & ME-HP (\%) \\
\hline Campesterol & 0.93 & 0.68 & 0,47 & 0.25 \\
Stigmasterol & 1.68 & 4.97 & 3.06 & 6.66 \\
E-Sitosterol & 2.18 & 3.58 & 2.55 & 4.44 \\
Epitaraxerol & - & - & - & - \\
E-Amyrin & 2.18 & 3.72 & 3.60 & 5.93 \\
D-Amyrin/Lupenone & 0.83 & 2.01 & 1.69 & 2.48 \\
Lupeol & 2.95 & 5.87 & 4.64 & 8.42 \\
Taraxerol acetate & - & - & - & - \\
Friedelin & 1.43 & 5.03 & 2.10 & - \\
Pseudotaraxasterol & 1.30 & 1.79 & 0.83 & 1.00 \\
Taraxasterol & 3.03 & 4.44 & 4.09 & 4.25 \\
11-oxours-12-ene & - & - & 0.15 & - \\
11-oxoolean-12-ene & - & - & - & - \\
E-Amyrin acetate & 13.06 & 14.30 & 13.79 & 11.39 \\
D-Amyrin acetate & 1.26 & 1.62 & 1.80 & 1.90 \\
Lupeol acetate & 13.94 & 12.41 & 19.60 & 16.04 \\
Taraxasterol acetate & 22.54 & 17.92 & 25.65 & 10.79 \\
\hline
\end{tabular}

DE: dichloromethanic extract; ME: methanolic extract; HP: hexanic phase; R: residue; -: traces. 
TABLE IV -Triterpenoid composition of M. cordifolia specimen collected from Campos de Jordão

\begin{tabular}{lcccc}
\hline Compounds & DE-R (\%) & DE-HP $(\%)$ & ME-R (\%) & ME-HP (\%) \\
\hline Campesterol & 0.87 & 0.32 & 0.41 & 0.27 \\
Stigmasterol & 2.47 & 2.96 & 3.36 & 2.57 \\
E-Sitosterol & 3.09 & 3.82 & 3.70 & 3.14 \\
Epitaraxerol & - & - & - & - \\
E-Amyrin & 5.66 & 7.95 & 10.45 & 10.35 \\
D-Amyrin/Lupenone & 1.63 & 2.61 & 2.19 & 3.02 \\
Lupeol & 2.49 & 4.62 & 2.55 & 4.48 \\
Taraxerol acetate & - & 0.47 & 0.42 & 0.79 \\
Friedelin & - & - & - & - \\
Pseudotaraxasterol & - & 0.62 & - & 0.45 \\
Taraxasterol & 2.57 & 2.30 & 4.63 & 3.04 \\
11-oxours-12-ene & - & - & - & 0.03 \\
11-oxoolean-12-ene & - & - & - & 0.03 \\
E-Amyrin acetate & 4.52 & 10.25 & 11.04 & 7.81 \\
D-Amyrin acetate & 0.31 & 1.71 & 1.91 & 1.00 \\
Lupeol acetate & 1.44 & 2.94 & 1.64 & 1.27 \\
Taraxasterol acetate & 15.68 & 20.67 & 23.18 & 15.72 \\
\hline
\end{tabular}

DE: dichloromethanic extract; ME: methanolic extract; HP: hexanic phase; R: residue; -: traces.

TABLE V - Triterpenoid composition of M. cordifolia specimen collected from Monte Verde

\begin{tabular}{lcccc}
\hline Compounds & DE-R $(\%)$ & DE-HP $(\%)$ & ME-R $(\%)$ & ME-HP (\%) \\
\hline Campesterol & 0.14 & - & 0.50 & 0.43 \\
Stigmasterol & 0.89 & 0.35 & 2.61 & 1.70 \\
E-Sitosterol & 2.47 & - & 6.96 & 4.01 \\
Epitaraxerol & - & - & 0.24 & - \\
E-Amyrin & 3.64 & 14.55 & 10.47 & 22.70 \\
D-Amyrin/Lupenone & 5.64 & 8.93 & 14.15 & 13.05 \\
Lupeol & 5.39 & 19.40 & 13.40 & 29.39 \\
Taraxerol acetate & - & - & 0.11 & - \\
Friedelin & - & - & - & - \\
Pseudotaraxasterol & 0.50 & 0.14 & 0.15 & 0.07 \\
Taraxasterol & 0.35 & - & 0.42 & 0.71 \\
11-oxours-12-ene & - & - & - & - \\
11-oxoolean-12-ene & - & 18.84 & - & - \\
E-Amyrin acetate & 19.09 & 0.67 & 11.56 & 0.85 \\
D-Amyrin acetate & 0.67 & 6.62 & - & - \\
Lupeol acetate & 15.71 & 0.22 & 16.79 & 1.05 \\
Taraxasterol acetate & 1.09 & 1.17 & 0.92 \\
\hline DE: dit & & & &
\end{tabular}

DE: dichloromethanic extract; ME: methanolic extract; HP: hexanic phase; R: residue; -: traces.

acetate, E-amyrin acetate and lupeol acetate were confirmed by co-injection of standards.

The steroids campesterol, stigmasterol, E-sitosterol, taraxasterol, pseudotaraxasterol and taraxasterol acetate were found in the extracts of all M. cordifolia specimens analysed. Epitaraxerol was just detected in Monte Ver- de specimen and taraxerol acetate in Monte Verde and Campos de Jordão specimens, both from forest vegetation.

Major triterpenoids found in the extracts are lupeol acetate, E-amyrin acetate and taraxasterol acetate (except for Monte Verde specimen). Friedelin was only observed 


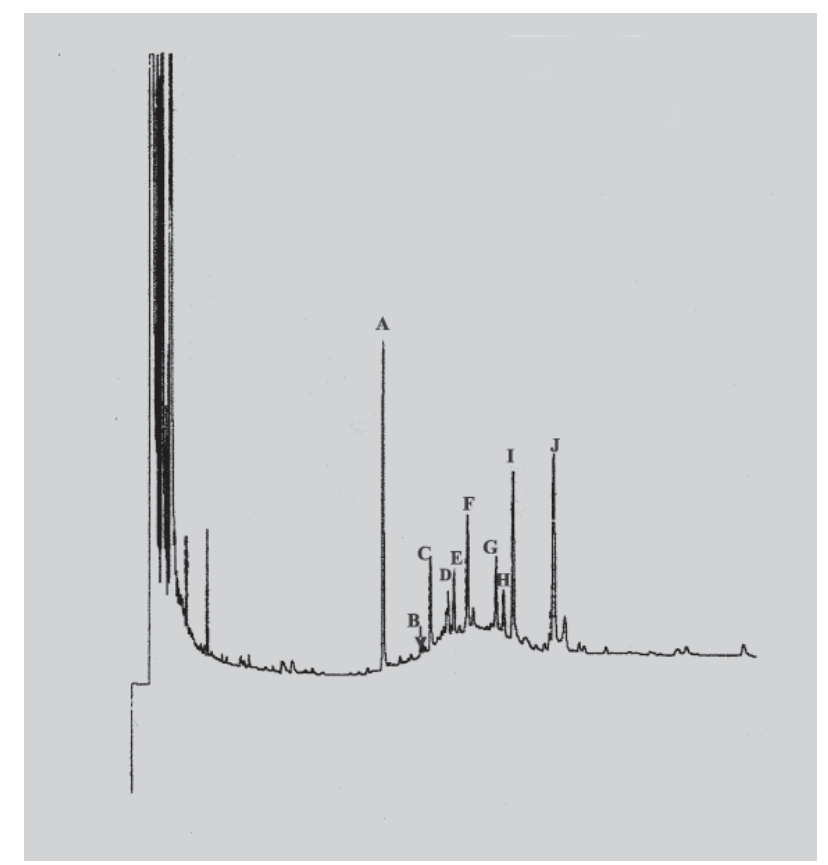

FIGURE 1 - GC chromatogram of triterpenoids from $M$. cordifolia from São Carlos- SP (DE-HP) using capilary colunm HP-1. (A) cholesterol (retention time: $14.182 \mathrm{~min}$.); (B) campesterol; (C) stigmasterol; (D) E-sitosterol; (E) Eamyrin; (F) D-amyrin/lupeol; (G) E-amyrin acetate; (H) taraxasterol; (I) D-amyrin acetate/lupeol acetate; (J) taraxasterol acetate.

in specimens collected from São Carlos and Ribeirão Preto, where the vegetation is cerrado (Brazilian savanna).

The use of two different capillary colunms ruled out the possibility of peak coincidence (Figure 1 and 2), but the coincidence of $\mathrm{D}$-amyrin and lupenone was excluded by analysis of the NMR ${ }^{13} \mathrm{C}$ spectral data (Olea, Roque, 1990), where it was just observed the lupenone presence.

\section{CONCLUSIONS}

The present work is the first study for triterpenoid chemistry in M. cordifolia collected from different locations and showed that there are no significant qualitative differences related to the presence of triterpenes and steroids. It can be concluded that all collected specimens of $M$. cordifolia presented similar constitution of triterpenoids, despite some possible differences in proportions.

\section{ACKNOWLEDGEMENTS}

The authors are grateful to Dr. Roberto Lourenço Esteves for botanical identification of plant material, to

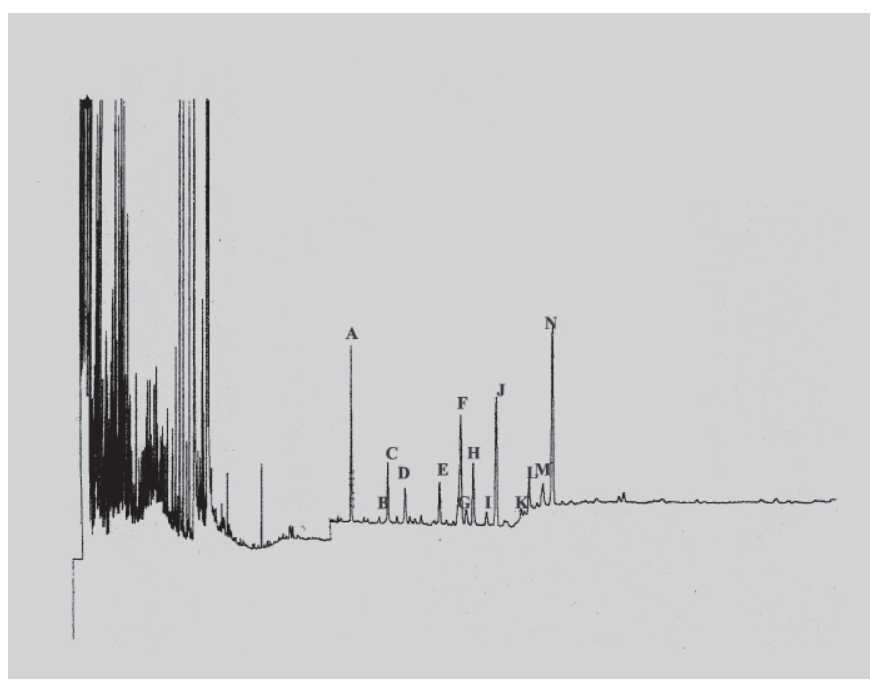

FIGURE 2 - GC chromatogram of triterpenoids from $M$. cordifolia from São Carlos- SP (DE-HP) using capilary colunm HP-50. (A) cholesterol (retention time: 14.182 min.); (B) campesterol; (C) stigmasterol; (D) E-sitosterol; (E) E-amyrin; (F) E-amyrin acetate; (G) D-amyrin/ lupenone; (H) lupeol; (I) D-amyrin acetate; (J) lupeol acetate; (K) pseudotaraxasterol; (L) taraxasterol; (M) friedelin; (N) taraxasterol acetate.

Dr. Noberto Peporine Lopes for collecting the specimens and to CNPq, CAPES and FAPESP for financial support.

\section{RESUMO}

\section{Análise comparativa de triterpenóides de Mikania cordifolia coletada em quatro locais diferentes}

A espécie Mikania cordifolia distribui-se por toda a América e é amplamente encontrada em quase todo o território brasileiro, onde é utilizada popularmente contra mordidas de serpentes. Extratos metanólicos e diclorometânicos preparados a partir e M. cordifolia Robinson coletadas em quatro locais diferentes do Brasil foram submetidos à extração líquido-líquido e os extratos hexânicos e residuos obtidos nesta etapa foram analisados para a pesquisa de triterpenóides por cromatografia em fase gasosa. Os espécimes coletados em Ribeirão Preto-SP e São Carlos-SP apresentaram os triterpenóides $\mathrm{E}$-amirina, lupeol, lupenona, acetato de D-amirina, acetato de E-amirina, acetato de lupeol, acetato de taraxasterol, campesterol e $\mathrm{E}$-sitosterol na suas composições. Além destes trierpenóides, o espécime de Campos de Jordão-SP apresentou 11-oxours-12eno, 11-oxoolean-12-eno e acetato de taraxerol e, o de Monte verde-MG, epitaraxerol e acetato de taraxerol. A 
friedelina foi observada apenas nas amostras de Ribeirão Preto-SP e São Carlos-SP.

UNITERMOS: Asteraceae. Mikania. Triterpenóides.

\section{REFERENCES}

ARIAS, A.R.; FERRO, E.; INCHAUSTI, A.; ASCURRA, M.; ACOSTA, N.; RODRIGUEZ, E.; FOURNET, A. Mutagenicity, insecticidal and trypanomicidal activity of some Paraguayan Asteraceae. J. Ethnopharmacol., Oxford, v. 45, p. 35 - 41, 1995.

BOEKER, P.; JAKUPOVIC, J.; BOHLMANN, F.; SCHMEDA-HIRSCHMANN, G. Germacra-1,10Z, 4Edien-12, 8 a-olides from Mikania micrantha. Planta Med., New York, v. 53, p.105-106, 1987.

BOHLMANN, F.; NATU, A. A.; MAHANTA, P. K. Neue diterpene und germacranolide aus Mikania Arten. Phytochemistry, Oxford, v. 17, n.3, p. 483 - 485, 1978.

CARIBE, J.; CAMPOS, J. M. Plantas que ajudam o homem. 5 ed. São Paulo: Cultrix, 1991.319p.

CASTRO, V.; JAKUPOVIC, J.; BOHLMANN, F. Germacranolides from Mikania species. Phytochemistry, Oxford, v. 28, p. 527-530, 1989.

CHAVES, J. S.; OLIVEIRA, D. C. R. Sesquiterpene Lactones and other constituents of Mikania hoehnei R. J. Braz. Chem. Soc., São Paulo, v. 14, n. 5, p. 734-737, 2003.

D’AGOSTINO, M.; DE SIMONE, F.; ZOLLO, F.; PIZZA, C. Constituents of Mikania cordifolia. Fitoterapia, Oxford,v. 62, n. 5, p.461, 1990.

FABBRI, H.; OLIVEIRA, D. C. R.; VICHNEWSKI, W.; HERZ, W. Diterpenes of Mikania lindberguii Baker. Biochem. Syst. Ecol., Oxford, v.25, n.6, p.563-564, 1997.

MOGHADASIAN, M. H. Pharmacological properties of plant sterols -In vivo and in vitro observations. Life Sci., Oxford, v. 67, n. 6, p. 605-615, 2000.

MORS, W. B.; NASCIMENTO, M. C.; PEREIRA, B. M. R.; PEREIRA, N. A. Plant natural products active against snake bite - the molecular approach. Phytochemistry, Oxford, v.55, n. 6, p. 627-642, 2000.
NASCIMENTO, A.M.; CHAVES, J.S.; ALBUQUERQUE, S.; OLIVEIRA, D.C.R. Trypanocidal properties of Mikania stipulacea and Mikania hoehnei isolated terpenoids. Fitoterapia, Oxford, v. 75, p. 381-384, 2004.

NASCIMENTO, A. M. ; OLIVEIRA, D. C. R. . Kaurene diterpenes and other chemical constituents from Mikania stipulaceae (M.Vahl) Willd. J. Braz. Chem. Soc., São Paulo, v. 12, n. 4, p. 552-555, 2001.

OLEA, R.S.G.; ROQUE, N.F. Análise de misturas de triterpenos por RMN de ${ }^{13}$ C. Quim. Nova, São Paulo, v. 13, n. $4,1990$.

OSVENA, Z.; VACHALKOVA, A.; HORVATHOVA, K. Taraxasterol and beta-sitosterol: new compouds with chemoprotective/chemopreventive effects - Minireview. Neoplasma, Brastislava, v. 51, n. 6, p. 407-414, 2004.

SALEEN, M.; AFAQ, F.; ADHAMI, V.M.; MUKHTAR, H. Lupeol modulates NF-kappaB and PI3K/Akt pathways and inhibits skin cancer in CD-1 mice. Oncogene, London, v. 23, n. 30, p. 5023-5214, 2004.

SULTANA, S.; SALEEN, M.; SHARMA, S.; KHAN, N. Lupeol, a triterpene, prevents free radical mediated macromolecular damage and alleviates benzoyl peroxide induced biochemical alterations in murine skin. Indian J. Exp. Biol., Nova Delhi, v. 41, n. 8, p. 827-831, 2003.

SCHINOR, E.C.; SALVADOR, M.J.; TURATTI, I.C.C.; ZUCCHI, O.L.A.D.; DIAS, D.A. Comparison of classical and ultrasound-assisted extractions of steroids and triterpenoids from three Chresta spp. Ultrason. Sonochem., Oxford, v. 11, p. 415-421, 2004.

SERRANO, S.M.; NOGAL, J.J.; DIAZ, M.R.A.; ESCARIO, J.A.; FERNÁNDEZ, A.R.; BARRIO, A.G. In vitro screening of American plant extracts on Trypanosoma cruzi and Trichomonas vaginalis. $J$. Ethnopharmacol., Oxford, v. 71, p. 101-107, 2000.

VENEZIANI, R. C. S.; OLIVEIRA, D. C. R. Constituents of Mikania glomerata. Biochem. Syst. Ecol., Oxford, v. 27, n. 1, p. 99-102, 1999.

Recebido para publicação em 22 de março de 2005 Aceito para publicação em 04 de agosto de 2006 